\title{
Evaluating the Therapeutic Role of Selected Active Compounds in Plumula Nelumbinis on Pulmonary Hypertension via Network Pharmacology and Experimental Analysis
}

\section{Xinghua Xiao}

Department of Pharmacy, Xiangya Hospital, Central South University

\section{Fangmei Luo}

Department of Pharmacy, Hunan Children's Hospital

\section{Minyi Fu}

Department of Pharmacy, Xiangya Hospital, Central South University

\section{Yueping Jiang}

Department of Pharmacy, Xiangya Hospital, Central South University

\section{Shao Liu}

Department of Pharmacy, Xiangya Hospital, Central South University

Bin Liu ( $\square$ liubin@csu.edu.cn )

Department of Pharmacy, Xiangya Hospital, Central South University

\section{Research Article}

Keywords: Pulmonary Hypertension, Network pharmacology, Molecular Docking, Plumula Nelumbinis, Alkaloids, PASMCs

Posted Date: January 27th, 2022

DOI: https://doi.org/10.21203/rs.3.rs-1270993/v1

License: (c) (i) This work is licensed under a Creative Commons Attribution 4.0 International License. Read Full License 


\section{Abstract}

Excessive proliferation of pulmonary artery smooth muscle cells (PASMCs) is one of the most critical factors leading to vascular remodeling in pulmonary hypertension $(\mathrm{PH})$. This study aimed to explore the effect and potential mechanism of Plumula Nelumbinis on PH by using network pharmacology and experimental analysis. The potential active components of Plumula Nelumbinis and their targets were obtained from TCMSP and PharmMapper databases. The PH-related targets were obtained from GeneCards, OMIM, DisGeNET, and TTD databases. Co-targets between Plumula Nelumbinis and PH were obtained by drawing Venn diagrams. The protein-protein interaction networks and compound-targetpathway networks were established using the STRING database and Cytoscape software. Moreover, the binding activities of core targets and essential active compounds were further validated by molecular docking. Finally, we further confirmed in vitro experiments that the main active components of Plumula Nelumbinis could inhibit the proliferation of PASMCs induced by hypoxia, as well as the increased expression of p-SRC and PIM1. Based on these observations, we conclude that multi-components (neferine, liensinine, and isoliensinine) in Plumula Nelumbinis can inhibit the hypoxia-induced proliferation of PASMCs by regulating the expression of p-SRC and PIM1, thereby delaying the development of $\mathrm{PH}$.

\section{Introduction}

Pulmonary hypertension $(\mathrm{PH})$ is a malignant cardiopulmonary vascular disease characterized by persistently elevated pulmonary arterial pressure ${ }^{1,2}$. Pulmonary vascular remodeling is the most basic pathological feature of $\mathrm{PH}$, involving processes such as the excessive proliferation of pulmonary artery smooth muscle cells (PASMCs), endothelial dysfunction, and extracellular matrix deposition ${ }^{3}$. Currently approved drugs for the treatment of $\mathrm{PH}$ mainly target three molecular pathways related to the pathogenesis of $\mathrm{PH}$ : including prostacyclins and prostacyclin-receptor agonists (such as epoprostenol, iloprost, selexipag, and treprostinil), endothelin receptor antagonists (such as ambrisentan, bosentan, and macitentan), nitric oxide pathway Agents (such as sildenafil and tadalafil) ${ }^{2}$. However, the above existing drug treatment usually only leads to an improvement in symptoms and cannot achieve the purpose of a radical cure. Therefore, it is urgent to find more safe and effective drugs to prevent and treat $\mathrm{PH}$.

Network pharmacology is a useful bioinformatics tool to reveal the complex biological network relationship between drugs, targets, and diseases by using high-throughput screening, network visualization, and analysis techniques ${ }^{4}$. In recent years, with the rise of network pharmacology, systematic research on the underlying mechanisms of traditional Chinese medicine (TCM) has been widely carried out, providing a foundation for the modernization of TCM ${ }^{5}$. From the perspective of TCM, the pathological mechanism of PH can be summarized as qi deficiency, blood stasis, and phlegm coagulation. Therefore, $\mathrm{PH}$ can be treated by nourishing the qi, invigorating the kidney and benefiting the lung, promoting blood circulation and removing blood stasis. Accumulating evidence suggests that active ingredients derived from TCM, such as magnolol ${ }^{6}$, magnesium lithospermate $B^{7}$, and resveratrol ${ }^{8}$, could 
delay the process of pulmonary vascular and right ventricular remodeling during $\mathrm{PH}^{9-11}$, which brings a promising future for the prevention and treatment of $\mathrm{PH}$.

Plumula Nelumbinis, a TCM derived from the dried young leaves and radicles of the mature seeds of the water lily plant Nelumbo nucifera Gaertn, could be used for medicine and food. It has multiple pharmacological activities such as cardiovascular protection, anti-oxidation, anti-inflammatory, and antitumor ${ }^{12}$. Recent studies have shown that alkaloids from Plumula Nelumbinis could attenuate vascular remodeling in spontaneously hypertensive rats ${ }^{13,14}$. However, its effect on vascular remodeling in $\mathrm{PH}$ is still unclear.

In this study, we first screened out the main active components and targets of Plumula Nelumbinis against PH through a network pharmacology strategy and then performed GO function and KEGG pathway enrichment analysis. Next, the interaction between the core compounds and targets was simulated by molecular docking. Finally, by establishing a hypoxia-induced PASMCs proliferation model, the prediction results were verified by in vitro experiments. The overall design of this study is shown in Fig. 1. The results of this study can further clarify the mechanism of Plumula Nelumbinis in the treatment of $\mathrm{PH}$ and provide a basis for the development of novel anti-PH drugs.

\section{Methods}

\section{Identification of the main active ingredient of Plumula Nelumbinis}

The active ingredients of Plumula Nelumbinis were obtained from the TCMSP database (Traditional Chinese Medicine Systems Pharmacology and Analysis Platform, https://old.tcmsp-e.com/tcmsp.php) ${ }^{34}$ and related literature ${ }^{12,35}$ by using search terms such as "Plumula Nelumbinis" or "Hindu Lotus Plumule". The chemical information (such as PubChem CID, 3D conformer, and canonical SMILES) and pharmacokinetic parameters of these active ingredients were collected from the PubChem (https://pubchem.ncbi.nlm.nih.gov/) and SwissADME (http://www.swissadme.ch/) database. The main active ingredients of Plumula Nelumbinis were screened according to the following conditions as described in the previous study ${ }^{36}$ : (1) The gastrointestinal absorption coefficient was set to High, which indicates that these compounds have good oral bioavailability and could be effectively absorbed; (2) The flexibility was evaluated by rotatable bonds: rotatable bonds $\leq 10$; (3) The polarity was evaluated by topological polar surface area (TPSA): $20 \AA^{2} \leq$ TPSA $\leq 140 \AA^{2}$; (4) The drug-likeness ability was evaluated by 5 different rule-based filters (Lipinski, Chose, Veber, Egan, and Muegge filter) and the bioavailability score: the bioavailability score $\geq 10 \%$. The potential targets of the main active ingredients of Plumula Nelumbinis were predicted through the PharmMapper database (http://www.lilabecust.cn/pharmmapper/submitfile.html, Normalized Fit Score $\geq 0.70)^{37}$. The potential targets were then identified and normalized through the UniProt database (https://www.uniprot.org/).

\section{Identification of PH-related targets}


The PH-related targets were obtained from GeneCards (https://www.genecards.org/), Online Mendelian Inheritance in Man (OMIM, https://omim.org/) ${ }^{38}$, DisGeNET (https://www.disgenet.org/) ${ }^{39}$, and Therapeutic Target Database (TTD, http://db.idrblab.net/ttd/) ${ }^{40}$ by using search terms such as "pulmonary hypertension" or "pulmonary arterial hypertension" (accessed on 21 Oct 2021). The above targets were summarized, de-duplicated, and then identified as PH-related targets.

\section{Construction of drug-target-disease and protein-protein interaction (PPI) Network}

The intersection between the potential targets of Plumula Nelumbinis and the PH-related targets was obtained by the VENNY online tool (https://bioinfogp.cnb.csic.es/tools/venny/index.html). The overlapping targets were considered as the potential therapeutic targets of Plumula Nelumbinis against $\mathrm{PH}$. The protein interaction relationships of the overlapped targets were acquired through the STRING database (https://cn.string-db.org/, Version 11.5) ${ }^{41}$. In brief, the overlapped targets were uploaded to the STRING database, and the species was limited to "Homo sapiens". Finally, the obtained PPI network information of the intersection targets was led to the Cytoscape software for visual analysis (https://cytoscape.org/, Version 3.8.2) ${ }^{42}$.

\section{GO and KEGG pathway enrichment analysis}

The Metascape online tool (https://metascape.org/) was applied for GO and KEGG enrichment analysis ${ }^{43}$. Briefly, the potential therapeutic targets of Plumula Nelumbinis against PH were uploaded to the Metascape platform. The filter thresholds of GO function and KEGG pathway analysis were set as the following conditions: (1) the species was set as "Homo sapiens"; (2) the minimum overlap value was set

to 3 ; (3) the $P$ cutoff value was set to 0.01 ; (4) the minimum enrichment value was set to 1.5 . The GO and KEGG enrichment analysis results were visualized through the ggplot2 package (Version 3.3.5) of the $R$ language (Version 4.0.2) ${ }^{44}$.

\section{Construction of the drug-target-pathway network}

The main component-target-pathway enrichment network was visualized using the Cytoscape software ${ }^{42}$. The supporting tool "Network Analyzer" of Cytoscape software was used to obtain the network topology parameters (such as degree, betweenness, and closeness) between the effective ingredients and targets. The top-ranked compounds and targets based on degree, betweenness, and closeness were defined as the core compounds and targets. These core compounds and targets were subsequently prepared for molecular docking.

\section{Molecular docking}

The binding situation of core compounds and targets was analyzed using AutoDock Vina software (https://vina.scripps.edu/, Version 1.1.2). Firstly, the three-dimensional crystal structures of the top 10 core target proteins were obtained from the Protein Data Bank (PDB, https://www.pdbus.org/) ${ }^{45}$. The PBD ID of these target proteins are as follows: proto-oncogene tyrosine-protein kinase Src (SRC, PDB ID: 
2SRC); serine/threonine-protein kinase PIM-1 (PIM1, PDBID:1XWS); transthyretin (TTR, PDB ID:1BZE); prothrombin (F2, PDB ID:2CN0); estrogen receptor-a (ESR1, PDB ID:5FQV); cyclophilin A (PPIA, PDB ID: 5LUD); mitogen-activated protein kinase 14 (MAPK14, PDB ID:2FST); phenylalanine-4-hydroxylase (PAH, PDB ID:5FII); $\beta$-secretase 1 (BACE1, PDB ID: 1TQF); endothelial nitric oxide synthase (NOS3, PDB ID:1M9M). Secondly, the 3D structures of the core compounds were obtained from the PubChem database (https://pubchem.ncbi.nlm.nih.gov/) and then were converted into PDB file format by using OPEN Babel software (http://openbabel.org/, Version 2.4.1) ${ }^{46}$. Finally, the molecular docking of core compounds and targets was performed using AutoDock Vina software ${ }^{47}$. The binding energy volcano map was drawn through the pheatmap package (Version 1.0.12) of the $R$ language. At the same time, the molecular docking results were visualized using the PyMol software (Version 2.2.0). The binding affinity value $<0 \mathrm{kcal} / \mathrm{mol}$ indicates that the compounds effectively bind to the targets.

\section{Chemicals and reagents}

The separation, extraction, identification of neferine, liensinine, isoliensinine, and the total alkaloids from Plumula Nelumbinis (TAPN) were performed as described in our previous study ${ }^{48-50}$. Dimethyl sulfoxide (DMSO) was purchased from Sigma-Aldrich (C6164, Darmstadt, Germany). High-glucose Dulbecco's modified Eagle's medium (DMEM) and fetal bovine serum (FBS) were purchased from Gibco (Thermo Fisher Scientific).

\section{Cell experiments}

The isolation and culture of PASMCs from the peripheral small pulmonary arteries of rats were performed as described in our previous study ${ }^{51}$. PASMCs at passages 3 to 6 were used for cell identification and the subsequent experiments. The hypoxia-induced PASMCs proliferation model was conducted as described in our previous study ${ }^{51}$. In order to evaluate the effect of the screened main active components of Plumula Nelumbinis on the proliferation of PASMCs induced by hypoxia, the following experiments were carried out: (1) the normoxia group, PASMCs were cultured in a normoxic incubator $\left(\mathrm{O}_{2} / \mathrm{CO}_{2} / \mathrm{N}_{2}=21 / 5 / 74\right)$ for $48 \mathrm{~h}$; (2) the hypoxia group, PASMCs were cultured in a hypoxia incubator $\left(\mathrm{O}_{2} / \mathrm{CO}_{2} / \mathrm{N}_{2}=3 / 5 / 92\right)$ for $48 \mathrm{~h}$; (3) the hypoxia plus drug intervention group, PASMCs were pretreated with different doses of drugs for $1 \mathrm{~h}$ and then treated with hypoxia for $48 \mathrm{~h}$, the initial drug doses for liensinine, liensinine, and isoliensinine were set at $1,2.5,5,10$, and $20 \mu \mathrm{M}$, respectively, while the initial doses of TAPN were $5,10,25,50$ and $100 \mathrm{ng} / \mathrm{ml}$, respectively. (4) the hypoxia plus vehicle group, PASMCs were pretreated with an equal volume of vehicle (DMSO, volume $\leq 1 / 1000$ of the total medium) for $1 \mathrm{~h}$ and then treated with hypoxia for $48 \mathrm{~h}$. At the end of the experiments, the PASMCs were collected for CCK8 detection and molecular analysis.

\section{Cell counting Kit-8 (CCK-8) detection}

Cell proliferation was detected by CCK-8 Assay Kit according to the protocol provided by the manufacturer (BA00208, Bioss, Beijing, China). Briefly, the PASMCs were inoculated into the 96-well 
plates and synchronized for $24 \mathrm{~h}$ after the cell density reached $50 \%$. After drug and hypoxia intervention, the CCK-8 solution was added to each well $(10 \mu \mathrm{L})$ and then incubated at $37^{\circ} \mathrm{C}$ for $2 \mathrm{~h}$ in the dark. The optical density (OD) value was detected at $450 \mathrm{~nm}$ with SpectraMax 190 (Molecular Devices, California, USA).

\section{Western blotting}

The PASMCs were homogenized in RIPA lysis buffer (Beyotime, Shanghai, China) with a protease and phosphatase inhibitor cocktail (Beyotime, Shanghai, China). Samples containing 30 40 $\mu \mathrm{g}$ of protein were separated by $10 \%$ SDS-PAGE gel and then transferred to polyvinylidene fluoride (PVDF) membranes (G.E. Healthcare, Germany). After blocking with 5\% BSA, the PVDF membranes were incubated overnight on a shaker at $4^{\circ} \mathrm{C}$ with the following primary antibodies: SRC (AF1831, 1:1000, Beyotime), p-SRC (AF5932, 1:1000, Beyotime), PIM1 (AF1807, 1:1000, Beyotime), eNOS (AF6792, 1:1000, Beyotime), and atubulin (sc-5286, 1:500, Santa Cruz). After incubation with horseradish peroxidase (HRP)-linked secondary antibody (Beyotime, Shanghai, China), the protein bands were imaged through Molecular Imager ChemiDoc XRS System (Bio-Rad, Philadelphia, USA). Densitometric quantification was performed by Image $\mathrm{J}(\mathrm{NIH}, \mathrm{USA})$. The a-tubulin served as a loading control.

\section{Statistical analysis}

All quantitative data were presented as the means \pm standard deviation (S.D.) and analyzed using SPSS 20.0 software (SPSS, Chicago, United States). One-way analysis of variance (ANOVA) was used to compare the means among different groups. $P \leq 0.05$ was considered statistically significant.

\section{Data availability}

The data supporting this study's findings are available from the corresponding author on reasonable request.

\section{Results}

\section{Screening of main active compounds from Plumula Nelumbinis}

More than 100 compounds have been reported to be isolated and identified from the Plumula Nelumbinis, including alkaloids (such as neferine, liensinine, and isoliensinine), flavonoids (such as quercetin, rutin, and kaempferol), volatile oil, and polysaccharides. A total of 92 possible active compounds were initially screened by matching with information from the PubChem database. Then the pharmacokinetic parameters of those compounds were obtained from the SwissADME database. Finally, we conducted a secondary screening of those compounds based on the gastrointestinal absorption rate, flexibility, polarity, and drug-likeness. A total of 46 main active ingredients of Plumula Nelumbinis were finally obtained, including 30 alkaloids, 7 flavonoids, and 9 other compounds (volatile oil or polysaccharides). The above 46 compounds were numbered as LZX1-LZX46, and relevant information is shown in Table 1. 
Table 1

The main active ingredients of Plumula Nelumbinis.

\begin{tabular}{|c|c|c|c|}
\hline Number & Pubchem name & $\begin{array}{l}\text { PubChem } \\
\text { ID }\end{array}$ & Class \\
\hline LZX1 & Neferine & 159654 & Alkaloids \\
\hline LZX2 & Liensinine & 160644 & Alkaloids \\
\hline LZX3 & Isoliensinine & 5274591 & Alkaloids \\
\hline LZX4 & Higenamine & 114840 & Alkaloids \\
\hline LZX5 & (S)-isococlaurine & 138319297 & Alkaloids \\
\hline LZX6 & (+)-N-Methylisococlaurine & 21817819 & Alkaloids \\
\hline LZX7 & Coclaurine & 160487 & Alkaloids \\
\hline LZX8 & (R)-N-Methylcoclaurine & 440595 & Alkaloids \\
\hline LZX9 & Lotusine & 5274587 & Alkaloids \\
\hline LZX10 & N-Nornuciferine & 12313579 & Alkaloids \\
\hline LZX11 & Nuciferine & 10146 & Alkaloids \\
\hline LZX12 & O-Nornuciferine & 197017 & Alkaloids \\
\hline LZX13 & Nornuciferidine & 183520 & Alkaloids \\
\hline LZX14 & Nelumboferine & 76046684 & Alkaloids \\
\hline LZX15 & Dauricine & 73400 & Alkaloids \\
\hline LZX16 & Pronuciferine & 200480 & Alkaloids \\
\hline LZX17 & Berberine & 2353 & Alkaloids \\
\hline LZX18 & (-)-Armepavine & 442169 & Alkaloids \\
\hline LZX19 & Lysicamine & 122691 & Alkaloids \\
\hline LZX20 & Anonaine & 160597 & Alkaloids \\
\hline LZX21 & Roemerine & 119204 & Alkaloids \\
\hline LZX22 & Liriodenine & 10144 & Alkaloids \\
\hline LZX23 & Thalifoline & 89048 & Alkaloids \\
\hline LZX24 & N-Methylcorydaldine & 303906 & Alkaloids \\
\hline LZX25 & 2-Methyl-1H-indole-3-carbaldehyde & 73166 & Alkaloids \\
\hline LZX26 & (R)-Norcoclaurine & 440988 & Alkaloids \\
\hline
\end{tabular}




\begin{tabular}{|llll|}
\hline Number & Pubchem name & $\begin{array}{l}\text { PubChem } \\
\text { ID }\end{array}$ & Class \\
\hline LZX27 & $\begin{array}{l}\text { 1alpha-(4-Methoxybenzyl)-2-methyl-6-methoxy-1,2,3,4- } \\
\text { tetrahydroisoquinoline }\end{array}$ & 132579667 & Alkaloids \\
\hline LZX28 & N-Methylisosalsoline & 40091 & Alkaloids \\
\hline LZX29 & O-Methylarmepavine & 821338 & Alkaloids \\
\hline LZX30 & 6,7-Dimethoxy-2-methyl-1,2,3,4-tetrahydroisoquinoline & 27694 & Alkaloids \\
\hline LZX31 & Quercetin & 5280343 & Flavonoids \\
\hline LZX32 & Isorhamnetin & 5281654 & Flavonoids \\
\hline LZX33 & Kaempferol & 5280863 & Flavonoids \\
\hline LZX34 & Diosmetin & 5281612 & Flavonoids \\
\hline LZX35 & Naringenin & 932 & Flavonoids \\
\hline LZX36 & Luteolin & 5280445 & Flavonoids \\
\hline LZX37 & Tricetin & 5281701 & Flavonoids \\
\hline LZX38 & 5-Hydroxymethylfurfural & 237332 & Others \\
\hline LZX39 & Linalool & 6549 & Others \\
\hline LZX40 & $\begin{array}{l}\text { (1,7,7-Trimethyl-2-bicyclo[2.2.1]heptanyl) 3-phenylprop-2- } \\
\text { enoate }\end{array}$ & 583021 & Others \\
\hline LZX41 & Gallic acid & 370 & Others \\
\hline LZX42 & Dibutyl phthalate & 5026 & Others \\
\hline LZX43 & Gamabufotalin & 5319081 & Others \\
\hline LZX44 & Loureirin A & 441491 & Others \\
\hline LZX45 & Methyl 4-hydroxycinnamate & & Others \\
\hline LZX46 & Propanethial S-oxide & 55803 & \\
\hline & Lidhers \\
\hline
\end{tabular}

Identification of potential targets of main active components from Plumula Nelumbinis

The potential targets of main active ingredients were obtained from the PharmMapper database. LZX46 (Propanethial S-oxide) was excluded because the predicted target of LZX46 was not available in the PharmMapper database. A total of 173 potential targets for the other 45 compounds were obtained after deduplication. The drug-target network is shown in Fig. 2. The red nodes represent alkaloids, the yellow nodes represent flavonoids, the purple nodes represent volatile oils or polysaccharides, and the green nodes represent the corresponding targets. 
Screening of potential targets of Plumula Nelumbinis against pulmonary hypertension

After summarizing and de-duplicating the targets, a total of $2326 \mathrm{PH}$-related targets were collected from GeneCards, OMIM, DisGeNET, and TTD databases. As shown in Fig. 3, a total of 60 common targets were obtained after the intersection of Plumula Nelumbinis-related targets and PH-related targets. These common targets were identified as potential therapeutic targets of Plumula Nelumbinis against PH.

\section{Construction of PPI network of anti-PH related targets of Plumula Nelumbinis}

The relationship between 60 intersection targets was obtained from the STRING database. The PPI network between those common targets was visualized using the Cytoscape software. As shown in Fig. 4, albumin (ALB), heat shock protein HSP 90-a (HSP90AA1), proto-oncogene tyrosine-protein kinase Src (SRC), caspase-3 (CASP3), estrogen receptor (ESR1), endothelial nitric oxide synthase (NOS3), vascular endothelial growth factor receptor 2 (KDR), mitogen-activated protein kinase 1 (MAPK1), plasminogen (PLG), peroxisome proliferator-activated receptor Y (PPARG), mitogen-activated protein kinase 14 (MAPK14), and annexin A5 (ANXA5) have high degree values in the PPI network, suggesting that these targets may be the critical targets for the main active components of Plumula Nelumbinis.

\section{GO and KEGG enrichment analysis}

GO enrichment analyses were conducted to confirm the intersection targets' function. GO function analysis results showed that the anti-PH effect of Plumula Nelumbinis might be closely related to a variety of biological processes (BP), including regulation of MAPK cascade (GO:0043408), wound healing (G0:0042060), reproductive structure development (G0:0048608), negative regulation of response to external stimulus (GO:0032102), cellular response to organic cyclic compound (GO:0071407), tube morphogenesis (G0:0035239), and protein phosphorylation (GO:0006468) (Fig. 5A and 5E). GO cellular components (CC) analysis terms mainly include cytoplasmic vesicle lumen (G0:0060205), membrane raft (GO:0045121), collagen-containing extracellular matrix (G0:0062023), lytic vacuole (G0:0000323), and receptor complex (GO:0043235) (Fig. 5B and 5F). GO molecular function (MF) analysis terms mainly include endopeptidase activity (GO:0004175) and protein serine/threonine/tyrosine kinase activity (G0:0004712) (Fig. 5C and 5G). KEGG pathway enrichment analysis indicated that the anti-PH effect of Plumula Nelumbinis might be achieved by regulating the following pathways: proteoglycans in cancer (hsa05205) and pathways in cancer (hsa05200) (Fig. 5D and 5H).

\section{Identification of core compounds and targets of Plumula Nelumbinis}

The compounds-targets-pathways network was constructed to clarify the core compounds and corresponding targets of Plumula Nelumbinis in PH treatment (Fig. 6). We first screened out nodes with degrees $\geq 25$ in the compound-target-pathway network as core targets, namely SRC, PIM1, TTR, F2, ESR1, PPIA, MAPK14, PAH, BACE1, and NOS3, respectively. Then the top 7 candidate core compounds were screened out according to the network topological parameters with the core targets, namely LZX1 (Neferine), LZX2 (Liensinine), LZX3 (Isoliensinine), LZX6 (N-Methylisococlaurine), LZX8 (N- 
Methylcoclaurine), LZX14 (Nelumboferine), and LZX18 (Armepavine). The above core compounds and targets were used for subsequent molecular docking verification.

\section{The molecule docking results for core compounds and targets}

In order to determine the possible binding interaction between core compounds and targets, molecular docking analysis was performed by using AutoDock Vina. The molecular docking results showed that the average binding energy between the core compounds and targets is $-7.03 \mathrm{kcal} / \mathrm{mol}$, indicating an effective binding between the compounds and targets. As we all know, the lower the binding energy score, the stronger the compound's ability to bind to the targets. By setting the binding energy threshold to -8.00 $\mathrm{kcal} / \mathrm{mol}$, we finally obtained three compounds (LZX1, LZX2, and LZX3) and three targets (SRC, PIM1, and NOS3) for subsequent cell experiments verification (Fig. 7A). The molecular docking pattern between the core compounds and targets is shown in Fig. 7B.

\section{Effect of Plumula Nelumbinis on hypoxia-induced hyperproliferation of PASMCs}

Based on the network pharmacology and molecular docking results, we further explored the effects and underlying mechanisms of the three core compounds on the hypoxia-induced proliferation of PASMCs. Since the core compounds (neferine, liensinine, and isoliensinine) belong to alkaloids, we also observed the effect of total alkaloids from Plumula Nelumbinis (TAPN) on cell proliferation. As shown in Fig. 8A-D, the hypoxia-induced proliferation of PASMCs was inhibited by TAPN, neferine, liensinine, and isoliensinine in a dose-dependent manner. Under normoxic conditions, the above drugs did not affect cell proliferation. The drug concentration (TAPN: $50 \mu \mathrm{g} / \mathrm{mL}$; neferine: $5 \mu \mathrm{M}$; liensinine and isoliensinine: 10 $\mu \mathrm{M})$ for subsequent experiments was determined based on the results of CCK-8.

Finally, we evaluated the underlying mechanisms of the 3 core compounds on PASMCs hyperproliferation. Compared to the control group, the protein expression of p-SRC and PIM1 in the hypoxia group was significantly up-regulated, and these increases were attenuated by TAPN, neferine, liensinine, and isoliensinine (Fig. 8E-G).

\section{Discussion}

In this study, the anti-PH effect and potential mechanisms of the main active components of Plumula Nelumbinis were evaluated by network pharmacology, molecular docking, and in vitro experimental verification. Firstly, we screened out the main active components of Plumula Nelumbinis against PH by using network pharmacology and molecular docking analysis. Then, by constructing a hypoxia-induced PASMCs proliferation model, we further revealed that the anti-PH mechanism of Plumula Nelumbinis might be achieved by inhibiting the activation of SRC and PIM1.

$\mathrm{PH}$ is a malignant cardiopulmonary vascular disease characterized by pulmonary vascular remodeling. With the continuous improvement of diagnosis and treatment technology in recent years, the five-year 
survival rate of patients with $\mathrm{PH}$ has reached $53.6 \%{ }^{15}$. Unfortunately, $\mathrm{PH}$ is still an intractable disease for the current therapies can only alleviate patients' symptoms and cannot effectively reverse the process of vascular remodeling. Therefore, it is an urgent clinical problem to seek more precise and effective interventions to prevent or treat PH. TCM has been used to treat PH through a multi-level and multi-target approach ${ }^{10}$. The rise of network pharmacology, characterized by "multiple compounds-multiple targetsmultiple signaling pathways", provides an effective way to systematically explore the internal mechanism of TCM in the treatment of $\mathrm{PH}^{4}$.

Plumula Nelumbinis is composed of various active ingredients, of which alkaloids are one of the main active ingredients. Recent studies showed that TAPN has the protective effect of inhibiting vascular remodeling in spontaneously hypertensive rats ${ }^{14}$. In addition, Jun et al. demonstrated that TAPN could inhibit the proliferation and migration of vascular smooth muscle cells, thereby inhibiting the carotid artery restenosis induced by balloon injury ${ }^{16}$. The above studies have shown that TAPN plays a cardiovascular protective effect by regulating the proliferation and migration of vascular smooth muscle cells. However, its effect on $\mathrm{PH}$ is still unclear. This study preliminarily screened 45 active components of Plumula Nelumbinis and 173 potential targets by consulting relevant literature and database information such as TCMSP, PubChem, SwissADME, and PharmMapper. Subsequently, 60 common targets were obtained by intersecting the potential active targets of Plumula Nelumbinis with PH-related targets, suggesting that Plumula Nelumbinis may delay the progression of $\mathrm{PH}$ by regulating multiple targets through multiple compounds.

$\mathrm{PH}$ is a complex disease involving multiple targets and pathways. In order to elucidate the functional and pathway enrichment of potential therapeutic targets in Plumula Nelumbinis, GO and KEGG enrichment analyses were performed using the Metascape platform. GO enrichment analysis showed that the anti-PH effect of Plumula Nelumbinis might be related to multiple biological processes and molecular functions, including regulation of MAPK cascade, wound healing, endopeptidase activity, and protein serine/threonine/tyrosine kinase activity. KEGG enrichment analysis revealed that its anti-PH effect might involve regulating cancer signaling pathways (hsa05205: proteoglycans in cancer; hsa05200: pathways in cancer; hsa05215: prostate cancer). It is worth noting that, comparable to cancer, $\mathrm{PH}$ is also a proliferative disease with high mortality and is thus known as the "cancer" of the cardiopulmonary vascular system. However, unlike cancer, which is caused by the excessive proliferation of cancer cells, $\mathrm{PH}$ is mainly caused by the excessive proliferation of vascular cells, especially the PASMCs in the media layer. Previous studies by ours and other groups demonstrated that neferine and isoliensinine have

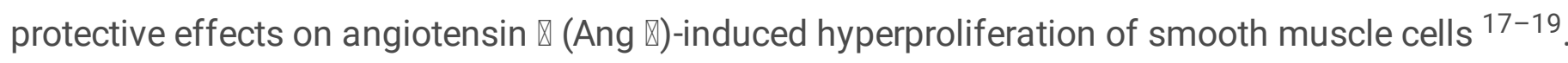
Based on the above reports and network pharmacology prediction, we speculate that the anti-PH effect of Plumula Nelumbinis may be related to the regulation of PASMCs hyperproliferation.

According to the compound-target-pathway network topology parameters, we obtained seven potential core compounds and ten core targets. The binding ability between the above compounds and targets was performed by molecular docking. Finally, three core compounds (LZX1, LZX2, and LZX3) and three core 
targets (SRC, PIM1, and NOS3) were identified for subsequent in vitro experiments by setting the binding energy threshold $\leq-8.0 \mathrm{kcal} / \mathrm{mol}$. SRC kinase, a proto-oncogene tyrosine-protein kinase, has been shown to mediate the occurrence and development of hypoxic $\mathrm{PH}$ by participating in the regulation of vasoconstriction, cell proliferation, and apoptosis ${ }^{20,21}$. Recently studies have proved that the effect of $\mathrm{SRC}$ in promoting hypoxic $\mathrm{PH}$ is closely related to the regulation of the activation of downstream proteins such as HIF-1a, HIF-2a, signal transducer and activator of transcription 3 (STAT3) and NADPH oxidase ${ }^{21}$. In addition, inhibition of SRC activation by drug intervention could effectively reverse vascular remodeling in experimental $\mathrm{PH}^{22,23}$, further clarifying the vital role of SRC in the promotion of PH. PIM1, a serine/threonine family kinase, has now been recognized as a novel biomarker and therapeutic target in the development of $\mathrm{PH}^{24}$. A large number of studies have demonstrated that PIM1 is overactivated in patients with $\mathrm{PH}$, preclinical animal models, or cell models ${ }^{25-27}$, and inhibition of PIM1 expression by drug intervention could also effectively delay the progression of $\mathrm{PH}^{26,28}$. NOS3, also known as eNOS, is mainly expressed in endothelial cells and could play a protective role in $\mathrm{PH}$ vascular remodeling by regulating nitric oxide synthesis ${ }^{29-31}$. The above studies show that SRC and PIM1 may participate in the development of PH by promoting the proliferation of PASMCs. On the contrary, NOS3 may alleviate the $\mathrm{PH}$ process by regulating the function of endothelial cells. Combined with the results of KEGG enrichment analysis, we preliminarily inferred that the anti-PH effect of Plumula Nelumbinis may be related to the inhibition of PASMCs proliferation by regulating the activation of SRC and PIM1.

Finally, we further clarified the anti-PH effect and potential mechanism of Plumula Nelumbinis in vitro cell experiments. Combined with $\mathrm{GO}$ enrichment analysis results, the biological process for Plumula Nelumbinis against PH involves response to hypoxia. To this end, we constructed a hypoxia-induced PASMCs proliferation model and then observed the effects of the three core compounds and TAPN on cell proliferation. Expectedly, the results from the present study found that TAPN, neferine, liensinine, and isoliensinine have a protective effect on hypoxia-induced PASMCs in a dose-dependent manner. Further results showed that the expression of PIM1 and p-SRC in PASMCs were significantly increased under hypoxic conditions; these phenomena were attenuated in the presence of TAPN, neferine, liensinine, and isoliensinine. These findings are consistent with our network pharmacology and molecular docking predictions, suggesting that the alkaloids of Plumula Nelumbinis may inhibit the proliferation of PASMCs by targeting SRC and PIM1, thereby achieving the purpose of treating PH. It is worth noting that we also detected the expression of eNOS in PASMCs after hypoxia treatment. However, the results showed that the basal expression of eNOS in PASMCs was deficient and challenging to detect. Further studies are needed to confirm the role of Plumula Nelumbinis on eNOS activity in pulmonary artery endothelial cells.

In addition to the hyperproliferation of PASMCs, hypoxia-induced contraction of PASMCs is also one of the crucial factors leading to $\mathrm{PH}^{32}$. Recently study found that neoliensinine, a novel benzylisoquinoline alkaloid extracted from Plumula Nelumbinis, has the effect of relaxing vascular smooth muscle cells ${ }^{33}$. The above study results show that the anti-PH effect of Plumula Nelumbinis may also be related to the inhibition of excessive contraction of PASMCs. However, follow-up studies are still needed to verify the effect of Plumula Nelumbinis on pulmonary vasoconstriction. 


\section{Conclusion}

In summary, this study demonstrated for the first time that multi-components (mainly alkaloids such as neferine, liensinine, and isoliensinine) in Plumula Nelumbinis could inhibit the hypoxia-induced proliferation of PASMCs by regulating the expression of multiple targets (SRC and PIM1). The above results can provide an experimental basis for the development of new drugs for $\mathrm{PH}$.

\section{Declarations}

\section{Acknowledgments}

This work was supported by the National Natural Science Foundation of China (No. 81703516 and No. 81703384), China Postdoctoral Science Foundation (No.2021M693575), and Natural Science Foundation of Hunan Province, China (No. 2019JJ50943, No.2021JJ80020, and No.2022JJ80084).

\section{Author contributions}

X.H.X. and F.M.L. participated in cell experiments and collected, analyzed, and processed experimental data. X.H.X. wrote the first draft. B.L., M.Y.F., Y.P.J., and S.L. were responsible for the design of the experiments, coordination of the project, and revision of the manuscript. All authors reviewed and approved the final version of the manuscript.

\section{Competing interests}

The authors declare no competing interests.

\section{Ethics statement}

The isolation and culture of primary PASMCs were derived from Sprague-Dawley (SD) rats. SD rats ( $180 \mathrm{~g}$ ) were provided by Hunan SJA Laboratory Animal Co.Ltd (No. SYXK (Xiang)2019-0004). All experimental animal protocols were approved by the Central South University Veterinary Medicine Animal Care and Use Committee. All animal recruitment, housing, treatment, and procedures were performed following the National Institutes of Health Guide for the Care and Use of Laboratory Animals. The animal studies followed the ARRIVE guidelines.

\section{Additional information}

Correspondence and requests for materials should be addressed to B.L.

\section{References}

1. Walter, K. Pulmonary Hypertension. JAMA 326, 1116, doi:10.1001/jama.2021.11054 (2021).

2. Poch, D. \& Mandel, J. Pulmonary Hypertension. Ann Intern Med 174, ITC49-ITC64, doi:10.7326/AITC202104200 (2021). 
3. Shimoda, L. A. Cellular Pathways Promoting Pulmonary Vascular Remodeling by Hypoxia.

Physiology (Bethesda, Md.) 35, 222-233, doi:10.1152/physiol.00039.2019 (2020).

4. Nogales, C. et al. Network pharmacology: curing causal mechanisms instead of treating symptoms. Trends Pharmacol Sci, doi:10.1016/j.tips.2021.11.004 (2021).

5. An, W. et al. Mechanisms of Rhizoma Coptidis against type 2 diabetes mellitus explored by network pharmacology combined with molecular docking and experimental validation. Sci Rep 11, 20849, doi:10.1038/s41598-021-00293-8 (2021).

6. Fu, M. et al. Magnolol Attenuates Right Ventricular Hypertrophy and Fibrosis in Hypoxia-Induced Pulmonary Arterial Hypertensive Rats Through Inhibition of the JAK2/STAT3 Signaling Pathway. Frontiers in pharmacology 12, 755077, doi:10.3389/fphar.2021.755077 (2021).

7. Li, $\mathrm{T}$. et al. Magnesium Lithospermate B Derived from Salvia miltiorrhiza Ameliorates Right Ventricle Remodeling in Pulmonary Hypertensive Rats via Inhibition of NOX/VPO1 Pathway. Planta Med 85, 708-718, doi:10.1055/a-0863-4741 (2019).

8. Mirhadi, E., Roufogalis, B. D., Banach, M., Barati, M. \& Sahebkar, A. Resveratrol: Mechanistic and therapeutic perspectives in pulmonary arterial hypertension. Pharmacol Res 163, 105287, doi:10.1016/j.phrs.2020.105287 (2021).

9. Zhang, J.-R. et al. Natural ingredients from Chinese materia medica for pulmonary hypertension. Chin J Nat Med 19, 801-814, doi:10.1016/S1875-5364(21)60092-4 (2021).

10. Xue, Z. et al. Traditional Herbal Medicine Discovery for the Treatment and Prevention of Pulmonary Arterial Hypertension. Frontiers in pharmacology 12, 720873, doi:10.3389/fphar.2021.720873 (2021).

11. Zhuang, P. et al. cAMP-PKA-CaMKII signaling pathway is involved in aggravated cardiotoxicity during Fuzi and Beimu Combination Treatment of Experimental Pulmonary Hypertension. Sci Rep 6, 34903, doi:10.1038/srep34903 (2016).

12. Chen, S. et al. Plumula Nelumbinis: A review of traditional uses, phytochemistry, pharmacology, pharmacokinetics and safety. J Ethnopharmacol 266, 113429, doi:10.1016/j.jep.2020.113429 (2021).

13. Wicha, P., Onsa-Ard, A., Chaichompoo, W., Suksamrarn, A. \& Tocharus, C. Vasorelaxant and Antihypertensive Effects of Neferine in Rats: An In Vitro and In Vivo Study. Planta Med 86, 496-504, doi:10.1055/a-1123-7852 (2020).

14. Li, Q. et al. Alkaloids from Nelumbinis Plumula (AFNP) ameliorate aortic remodeling via RhoA/ROCK pathway. Biomed Pharmacother 112, 108651, doi:10.1016/j.biopha.2019.108651 (2019).

15. Gall, H. et al. The Giessen Pulmonary Hypertension Registry: Survival in pulmonary hypertension subgroups. J Heart Lung Transplant 36, 957-967, doi:10.1016/j.healun.2017.02.016 (2017).

16. Jun, M. Y. et al. Alkaloid rich fraction from Nelumbo nucifera targets VSMC proliferation and migration to suppress restenosis in balloon-injured rat carotid artery. Atherosclerosis 248, 179-189, doi:10.1016/j.atherosclerosis.2016.03.020 (2016). 
17. Zheng, L., Cao, Y., Liu, S., Peng, Z. \& Zhang, S. Neferine inhibits angiotensin Il-induced rat aortic smooth muscle cell proliferation predominantly by downregulating fractalkine gene expression. Exp Ther Med 8, 1545-1550 (2014).

18. Li, X.-c. et al. Neferine inhibits angiotensin Il-stimulated proliferation in vascular smooth muscle cells through heme oxygenase-1. Acta Pharmacol Sin 31, 679-686, doi:10.1038/aps.2010.57 (2010).

19. Xiao, J. H., Zhang, Y. L., Feng, X. L., Wang, J. L. \& Qian, J. Q. Effects of isoliensinine on angiotensin IIinduced proliferation of porcine coronary arterial smooth muscle cells. J Asian Nat Prod Res 8, 209216 (2006).

20. Norton, C. E. et al. Augmented Pulmonary Vasoconstrictor Reactivity after Chronic Hypoxia Requires Src Kinase and Epidermal Growth Factor Receptor Signaling. American journal of respiratory cell and molecular biology 62, 61-73, doi:10.1165/rcmb.2018-01060C (2020).

21. Gao, Y. \& Raj, J. U. Src and Epidermal Growth Factor Receptor: Novel Partners in Mediating Chronic Hypoxia-induced Pulmonary Artery Hypertension. American journal of respiratory cell and molecular biology 62, 5-7, doi:10.1165/rcmb.2019-0230ED (2020).

22. Pullamsetti, S. S. et al. Role of Src tyrosine kinases in experimental pulmonary hypertension. Arterioscler Thromb Vasc Biol 32, 1354-1365, doi:10.1161/ATVBAHA.112.248500 (2012).

23. Liu, P. et al. Inhibition of Src activation reverses pulmonary vascular remodeling in experimental pulmonary arterial hypertension via Akt/mTOR/HIF-1<alpha> signaling pathway. Exp Cell Res 380, 36-46, doi:10.1016/j.yexcr.2019.02.022 (2019).

24. Satoh, K., Kikuchi, N. \& Shimokawa, H. PIM1 (Provirus Integration Site For Moloney Murine Leukemia Virus) as a Novel Biomarker and Therapeutic Target in Pulmonary Arterial Hypertension: Another Evidence for Cancer Theory. Arterioscler Thromb Vasc Biol 40, 500-502, doi:10.1161/ATVBAHA.120.313975 (2020).

25. Paulin, R. et al. Signal transducers and activators of transcription-3/pim 1 axis plays a critical role in the pathogenesis of human pulmonary arterial hypertension. Circulation 123, 1205-1215, doi:10.1161/CIRCULATIONAHA.110.963314 (2011).

26. Braga, C. L. et al. Niclosamide attenuates lung vascular remodeling in experimental pulmonary arterial hypertension. European journal of pharmacology 887, 173438, doi:10.1016/j.ejphar.2020.173438 (2020).

27. $\mathrm{Ge}, \mathrm{X}$. et al. Hypoxia-activated platelets stimulate proliferation and migration of pulmonary arterial smooth muscle cells by phosphatidylserine/LOX-1 signaling-impelled intercellular communication. Cell Signal 87, 110149, doi:10.1016/j.cellsig.2021.110149 (2021).

28. Lampron, M.-C. et al. PIM1 (Moloney Murine Leukemia Provirus Integration Site) Inhibition Decreases the Nonhomologous End-Joining DNA Damage Repair Signaling Pathway in Pulmonary Hypertension. Arterioscler Thromb Vasc Biol 40, 783-801, doi:10.1161/ATVBAHA.119.313763 (2020).

29. Somani, A. et al. Blood outgrowth endothelial cells overexpressing eNOS mitigate pulmonary hypertension in rats: a unique carrier cell enabling autologous cell-based gene therapy. Transl Res 210, 1-7, doi:10.1016/j.trsl.2019.04.005 (2019). 
30. Hua, C. et al. Apple polyphenol relieves hypoxia-induced pulmonary arterial hypertension via pulmonary endothelium protection and smooth muscle relaxation: In vivo and in vitro studies. Biomed Pharmacother 107, 937-944, doi:10.1016/j.biopha.2018.08.080 (2018).

31. Zhou, Y. et al. Quaternary ammonium salt of U50,488H elicits protective effects against hypoxic pulmonary hypertension. European journal of pharmacology 832, 129-137, doi:10.1016/j.ejphar.2018.05.025 (2018).

32. Sommer, N., Strielkov, I., Pak, O. \& Weissmann, N. Oxygen sensing and signal transduction in hypoxic pulmonary vasoconstriction. Eur Respir J 47, 288-303, doi:10.1183/13993003.00945-2015 (2016).

33. Yang, G.-M. et al. ITRAQ-Based Proteomics Analysis Reveals the Effect of Neoliensinine on KClInduced Vascular Smooth Muscle Contraction by Inhibiting Regulatory Light Chain Phosphorylation. Frontiers in pharmacology 10,979, doi:10.3389/fphar.2019.00979 (2019).

34. Ru, J. et al. TCMSP: a database of systems pharmacology for drug discovery from herbal medicines. J Cheminform 6, 13, doi:10.1186/1758-2946-6-13 (2014).

35. Jiang, Y., Zi, W., Pei, Z. \& Liu, S. Characterization of polysaccharides and their antioxidant properties from Plumula nelumbinis. Saudi Pharm J 26, 656-664, doi:10.1016/j.jsps.2018.02.026 (2018).

36. Daina, A., Michielin, O. \& Zoete, V. SwissADME: a free web tool to evaluate pharmacokinetics, druglikeness and medicinal chemistry friendliness of small molecules. Sci Rep 7, 42717, doi:10.1038/srep42717 (2017).

37. Wang, X. et al. PharmMapper 2017 update: a web server for potential drug target identification with a comprehensive target pharmacophore database. Nucleic Acids Res 45, W356-W360, doi:10.1093/nar/gkx374 (2017).

38. Amberger, J. S., Bocchini, C. A., Scott, A. F. \& Hamosh, A. OMIM.org: leveraging knowledge across phenotype-gene relationships. Nucleic Acids Res 47, D1038-D1043, doi:10.1093/nar/gky1151 (2019).

39. Pinero, J., Sauch, J., Sanz, F. \& Furlong, L. I. The DisGeNET cytoscape app: Exploring and visualizing disease genomics data. Comput Struct Biotechnol J 19, 2960-2967, doi:10.1016/j.csbj.2021.05.015 (2021).

40. Zhou, Y. et al. Therapeutic target database update 2022: facilitating drug discovery with enriched comparative data of targeted agents. Nucleic Acids Res, doi:10.1093/nar/gkab953 (2021).

41. Szklarczyk, D. et al. The STRING database in 2017: quality-controlled protein-protein association networks, made broadly accessible. Nucleic Acids Res 45, D362-D368, doi:10.1093/nar/gkw937 (2017).

42. Shannon, P. et al. Cytoscape: a software environment for integrated models of biomolecular interaction networks. Genome Res 13, 2498-2504, doi:10.1101/gr.1239303 (2003).

43. Zhou, Y. et al. Metascape provides a biologist-oriented resource for the analysis of systems-level datasets. Nat Commun 10, 1523, doi:10.1038/s41467-019-09234-6 (2019).

44. Wickham, H. et al. ggplot2: Elegant Graphics for Data Analysis. Vol. Springer-Verlag New York (Springer-Verlag New York, 2016). 
45. Burley, S. K. et al. RCSB Protein Data Bank: powerful new tools for exploring 3D structures of biological macromolecules for basic and applied research and education in fundamental biology, biomedicine, biotechnology, bioengineering and energy sciences. Nucleic Acids Res 49, D437-D451, doi:10.1093/nar/gkaa1038 (2021).

46. O'Boyle, N. M. et al. Open Babel: An open chemical toolbox. J Cheminform 3, 33, doi:10.1186/17582946-3-33 (2011).

47. Trott, O. \& Olson, A. J. AutoDock Vina: improving the speed and accuracy of docking with a new scoring function, efficient optimization, and multithreading. J Comput Chem 31, 455-461, doi:10.1002/jcc.21334 (2010).

48. Jiang, Y., Liu, R., Liu, M., Yi, L. \& Liu, S. An integrated strategy to rapidly characterize non-targeted benzylisoquinoline alkaloids from Plumula nelumbinis ethanol extract using UHPLC/Q-orbitrap HRMS. International Journal of Mass Spectrometry 432, 26-35, doi:10.1016/j.ijms.2018.06.002 (2018).

49. Liu, S., Wang, B., Li, X. Z., Qi, L. F. \& Liang, Y. Z. Preparative separation and purification of liensinine, isoliensinine and neferine from seed embryo of Nelumbo nucifera GAERTN using high-speed countercurrent chromatography. J Sep Sci 32, 2476-2481, doi:10.1002/jssc.200800766 (2009).

50 . Chen, J. et al. Neferine and lianzixin extracts have protective effects on undifferentiated caffeinedamaged PC12 cells. BMC Complement Med Ther 20, 76, doi:10.1186/s12906-020-2872-2 (2020).

51. Li, T. et al. Magnesium lithospermate B prevents phenotypic transformation of pulmonary arteries in rats with hypoxic pulmonary hypertension through suppression of NADPH oxidase. Eur J Pharmacol 847, 32-41, doi:10.1016/j.ejphar.2019.01.020 (2019).

\section{Figures}




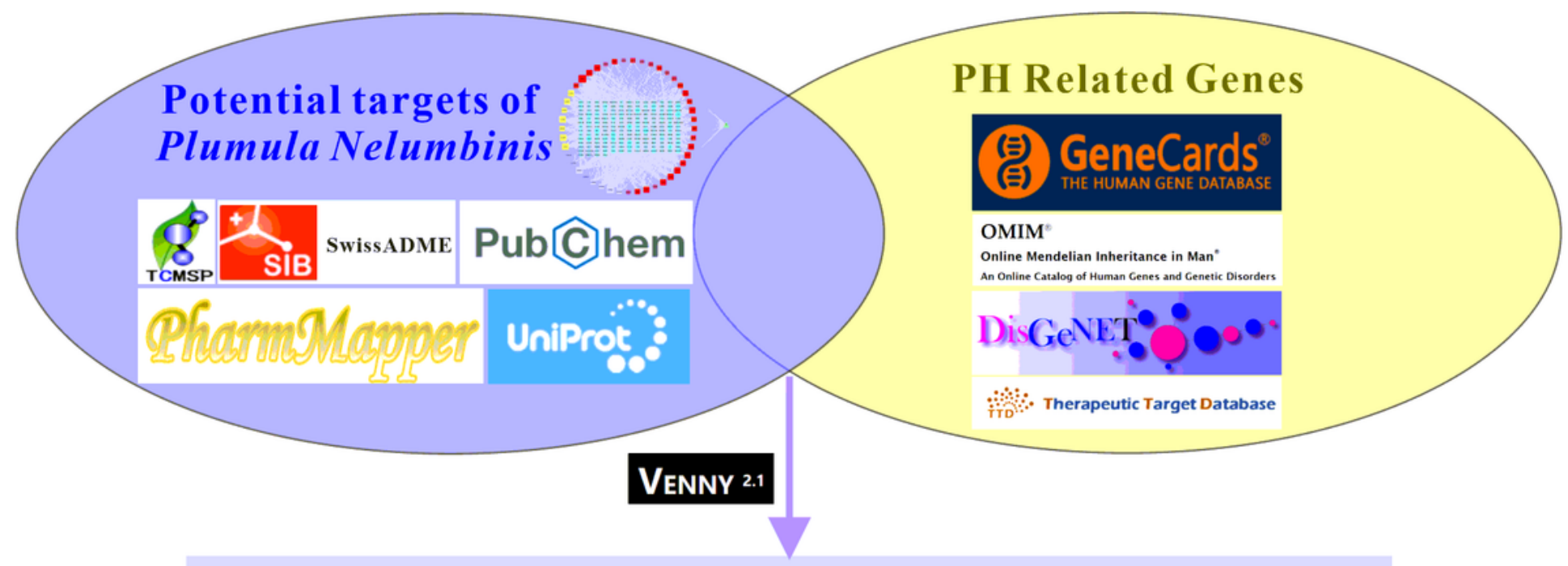

\section{Potential Therapeutic Targets of Plumula Nelumbinis Against PH}
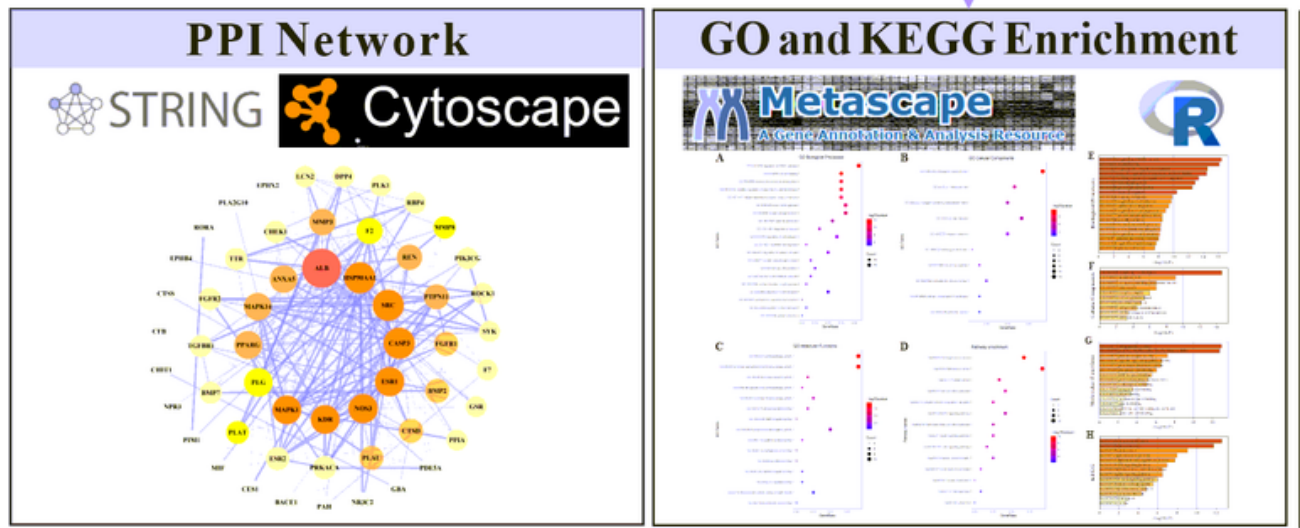

Drug-Targets-Pathway Network
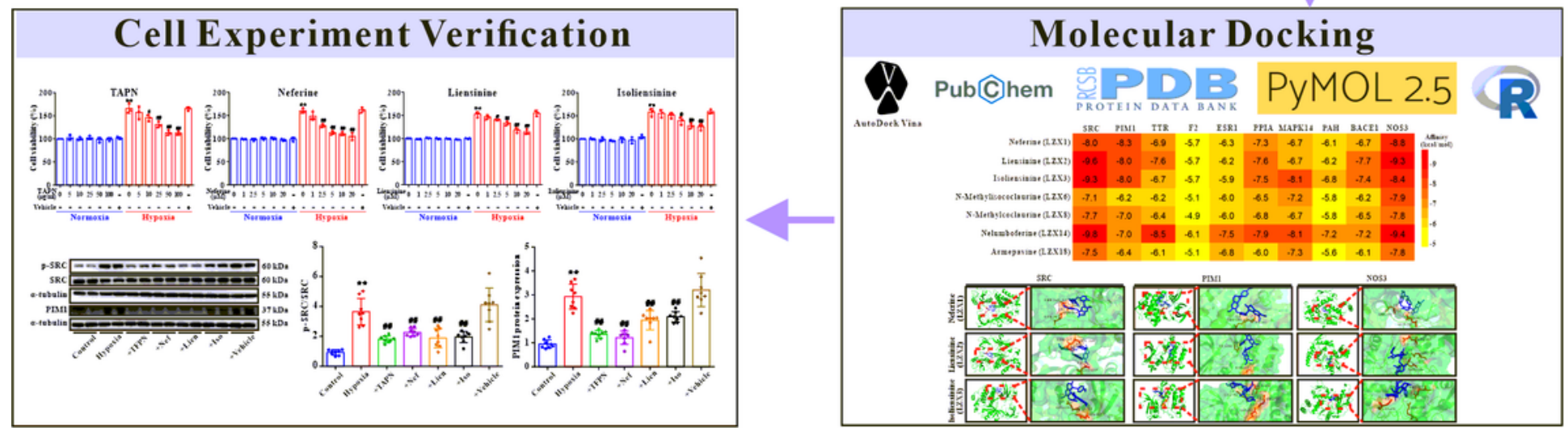

Figure 1

The network pharmacology workflow of Plumula Nelumbinis against pulmonary hypertension. $\mathrm{PH}$, pulmonary hypertension; PPI, protein-protein interactions; GO, gene ontology; KEGG, Kyoto encyclopedia of genes and genomes. 


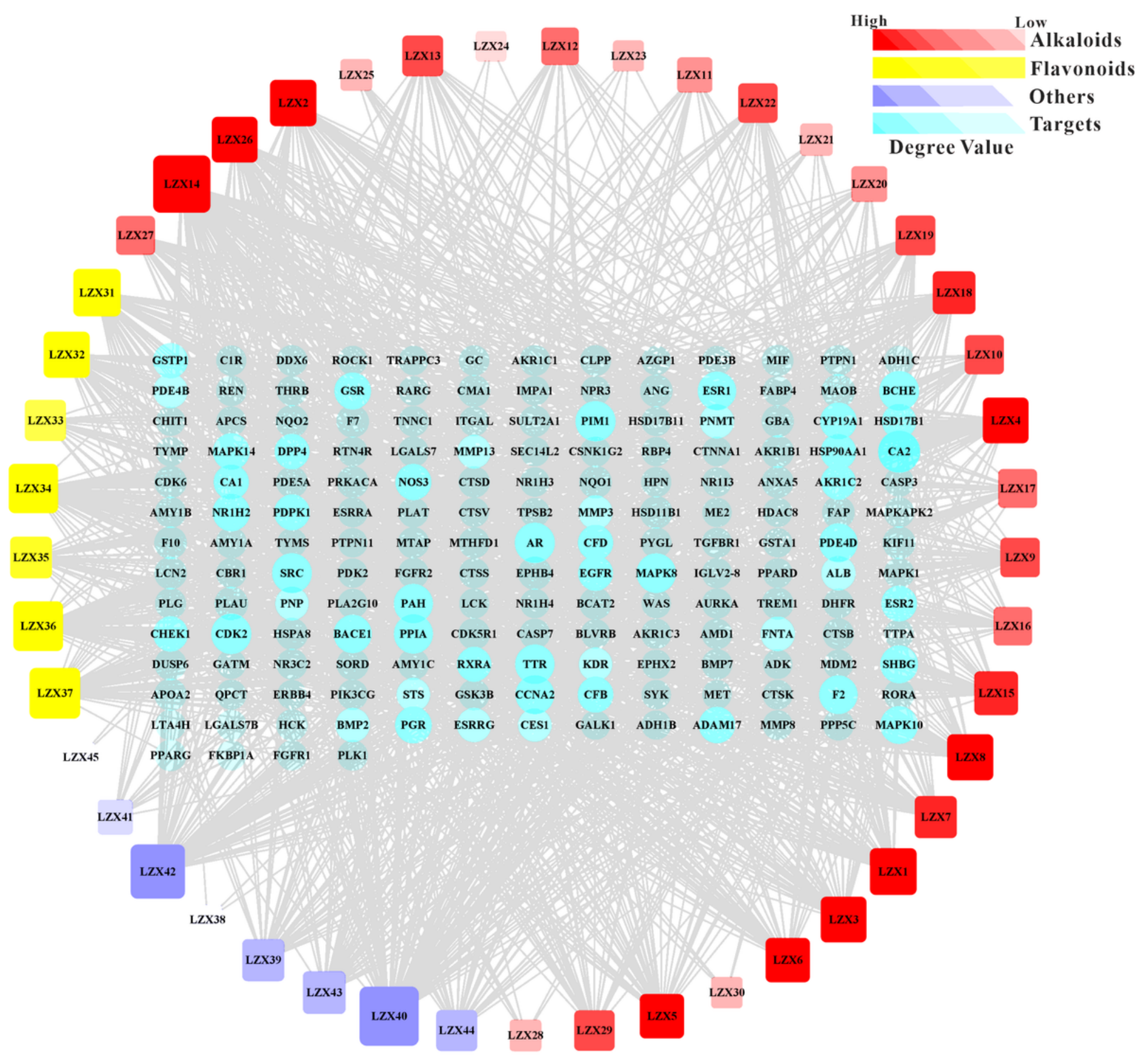

\section{Figure 2}

Potential targets of active ingredients in Plumula Nelumbinis. The circles represent targets (173 in total). The squares represent the active compounds from Plumula Nelumbinis (45 in total), including 30 alkaloids (marked in red), seven flavonoids (marked in yellow), and nine other compounds (volatile oils or polysaccharides, marked in purple). The importance of the compound or target in the network was evaluated by the degree value. The darker the square/circle color, the higher the degree value of the corresponding compound/ target, indicating that the compound or target is more critical in the network. 


\section{Potential targets of Plumula Nelumbinis}

\section{PH Related Genes}

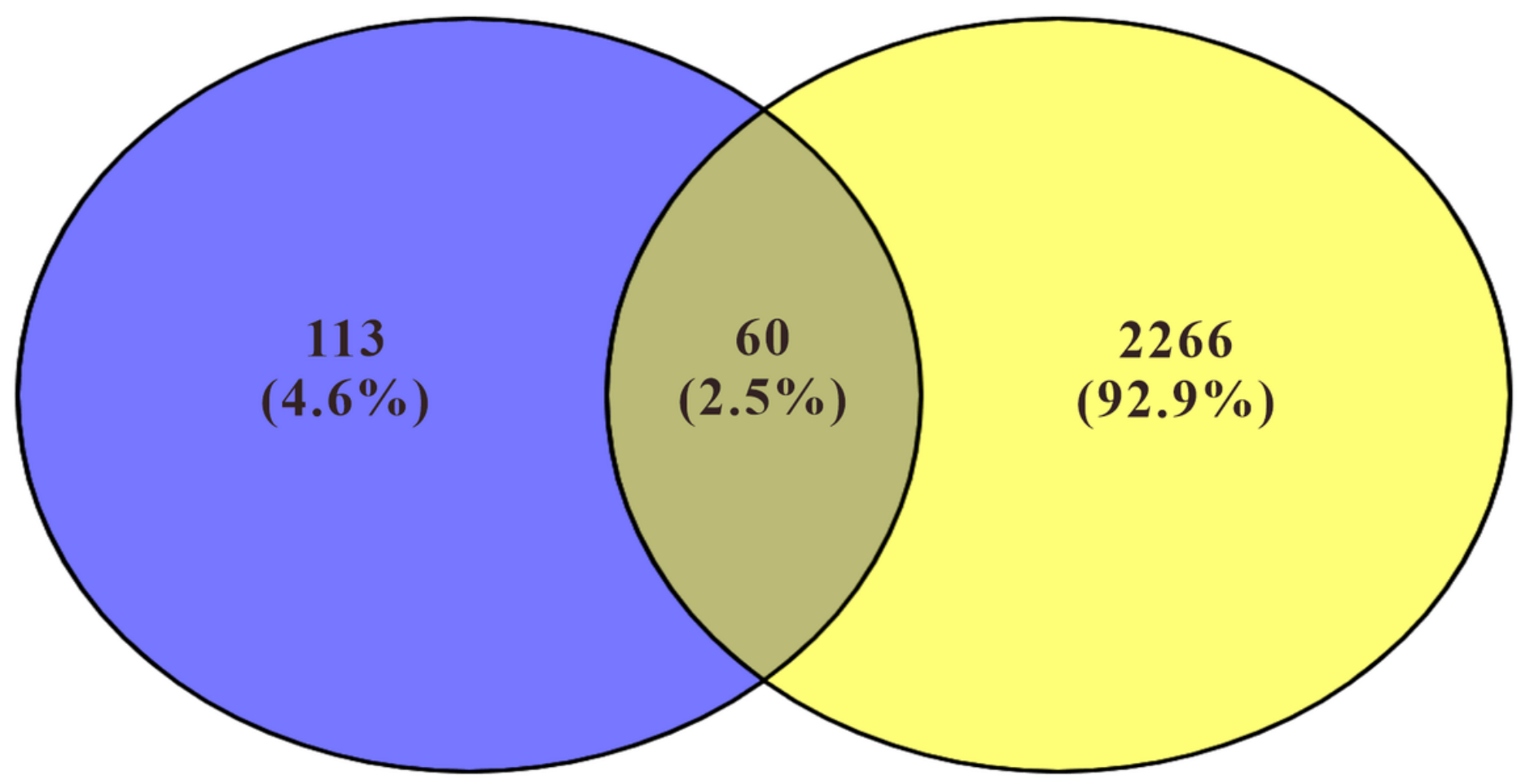

Figure 3

Venn diagram of potential targets of the main active components of Plumula Nelumbinis against PH. A total of 173 potential targets of the main active components of Plumula Nelumbinis were obtained by using the PharmMapper database. A total of 2326 pulmonary hypertension-related targets were obtained using GeneCards, OMIM, DisGeNET, and TTD databases. The intersection of the potential targets of Plumula Nelumbinis and PH-related targets was obtained by using the VENNY 2.1 online tool, which was identified as the potential targets of Plumula Nelumbinis against $\mathrm{PH}$. 


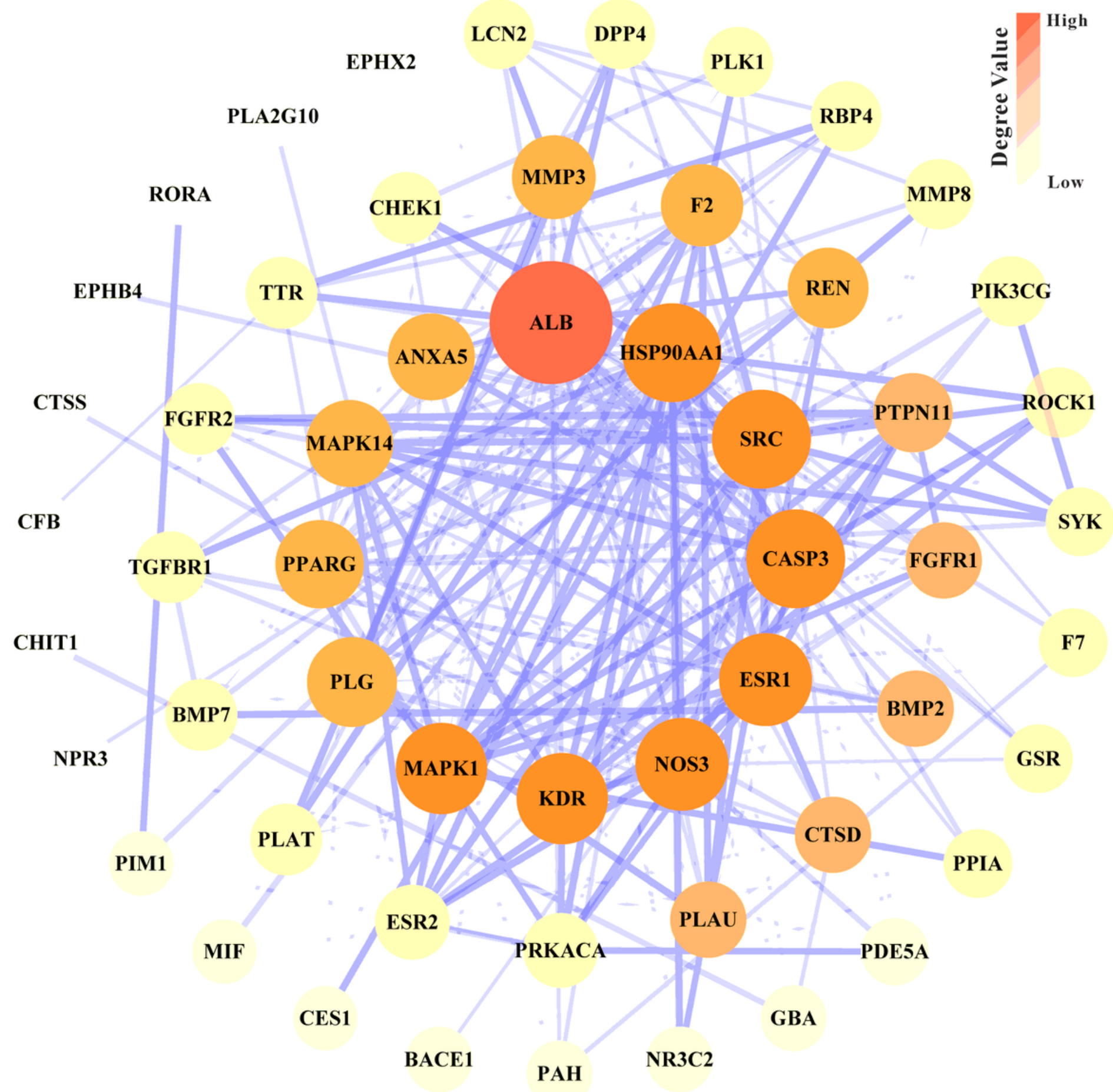

Figure 4

PPI network diagram of potential targets of Plumula Nelumbinis against PH. 
A

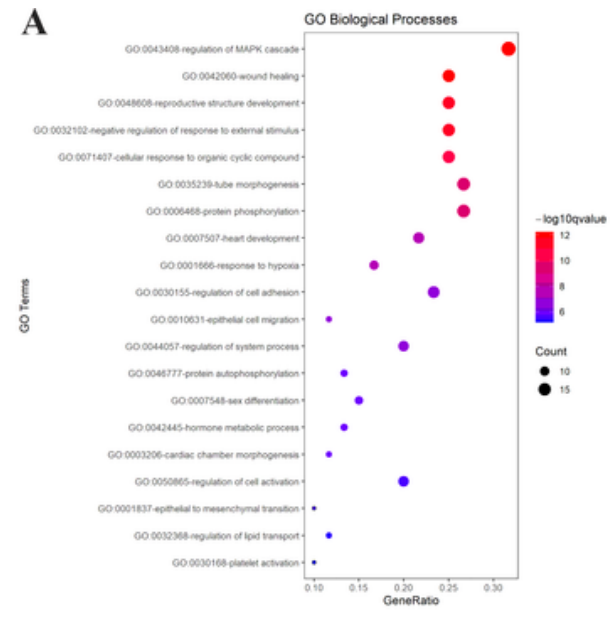

C

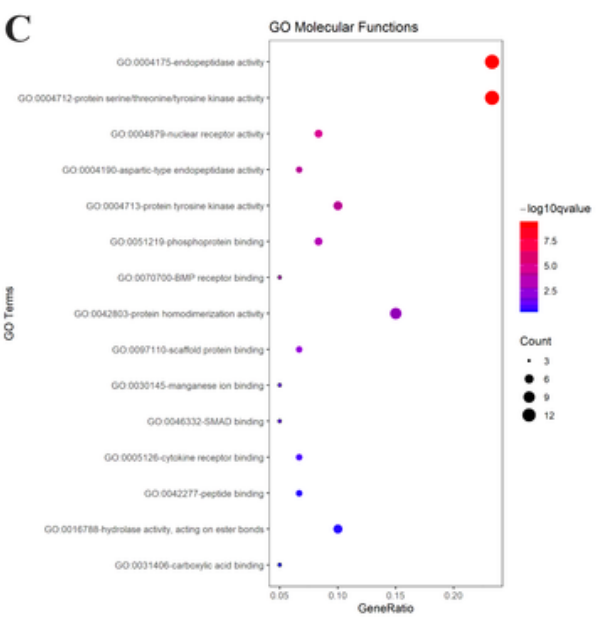

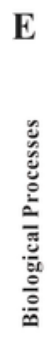
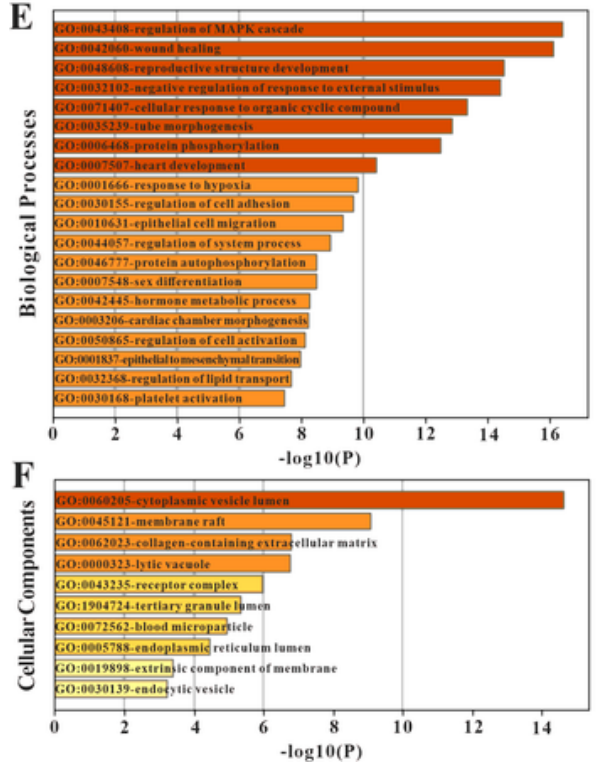

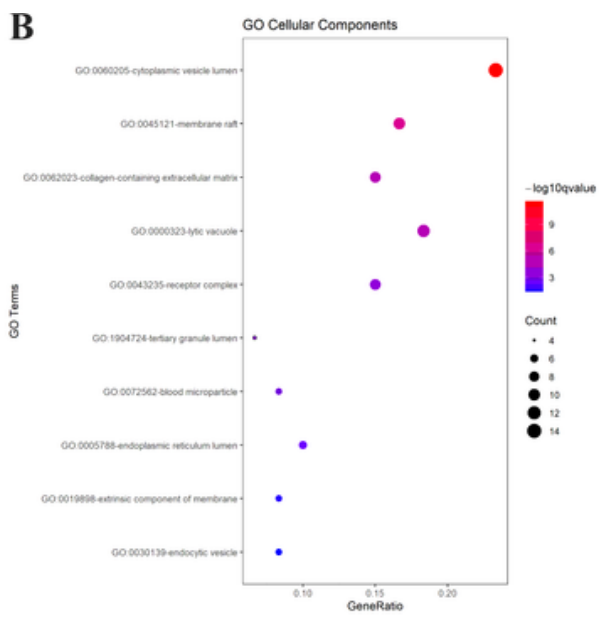

D
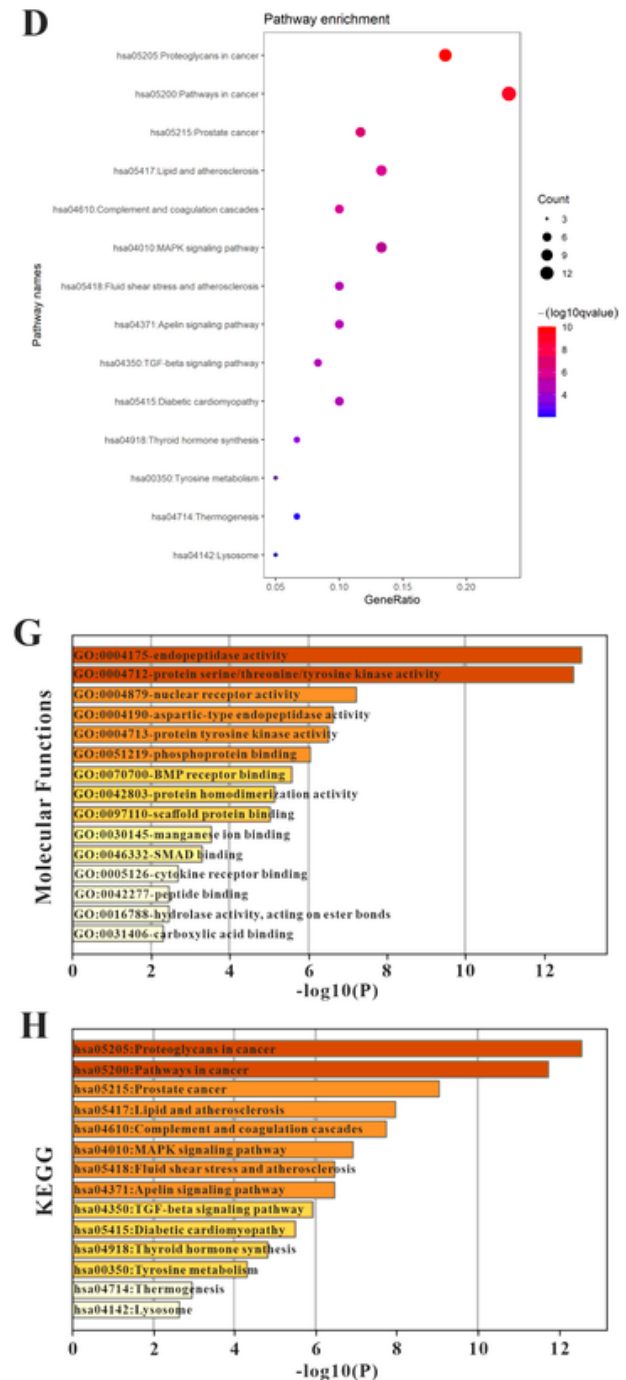

Figure 5

GO and KEGG enrichment analysis of the potential targets of Plumula Nelumbinis against PH. (A, E) GO biological processes analysis. (B, F) GO cellular components analysis. (C, G) GO molecular functions analysis. (D, H) KEGG pathway enrichment analysis. 


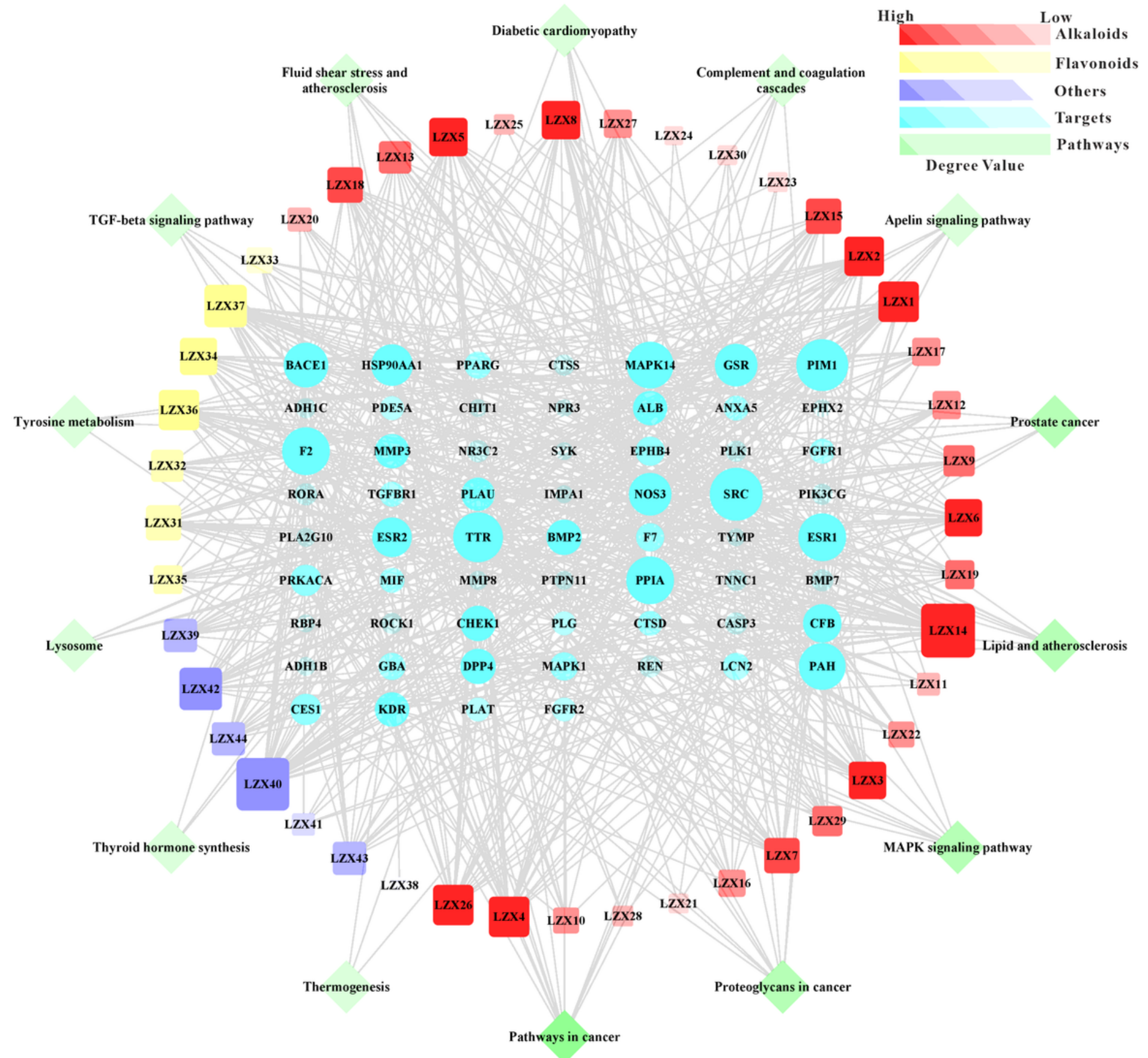

\section{Figure 6}

Compounds-targets-pathways network analysis diagram. 


\begin{tabular}{|c|c|c|c|c|c|c|c|c|c|c|c|}
\hline & SRC & PIM1 & TTR & F2 & ESR1 & PPIA & MAPK14 & PAH & BACE1 & NOS3 & \\
\hline Neferine (LZX1) & -8.0 & -8.3 & -6.9 & -5.7 & -6.3 & -7.3 & -6.7 & -6.1 & -6.7 & -8.8 & $\begin{array}{c}\text { Affinity } \\
(\mathrm{kcal} / \mathrm{mol})\end{array}$ \\
\hline Liensinine (LZX2) & -9.6 & -8.0 & -7.6 & -5.7 & -6.2 & -7.6 & -6.7 & -6.2 & -7.7 & -9.3 & -9 \\
\hline Isoliensinine (LZX3) & -9.3 & -8.0 & -6.7 & -5.7 & -5.9 & -7.5 & -8.1 & -6.8 & -7.4 & -8.4 & \\
\hline -Methylisococlaurine (LZX6) & -7.1 & -6.2 & -6.2 & -5.1 & -6.0 & -6.5 & -7.2 & -5.8 & -6.2 & -7.9 & \\
\hline N-Methylcoclaurine (LZX8) & -7.7 & -7.0 & -6.4 & -4.9 & -6.0 & -6.8 & -6.7 & -5.8 & -6.5 & -7.8 & \\
\hline Nelumboferine (LZX14) & -9.8 & -7.0 & -8.5 & -6.1 & -7.5 & -7.9 & -8.1 & -7.2 & -7.2 & -9.4 & \\
\hline Armepavine (LZX18) & -7.5 & -6.4 & -6.1 & -5.1 & -6.8 & -6.0 & -7.3 & -5.6 & -6.1 & -7.8 & \\
\hline
\end{tabular}

B
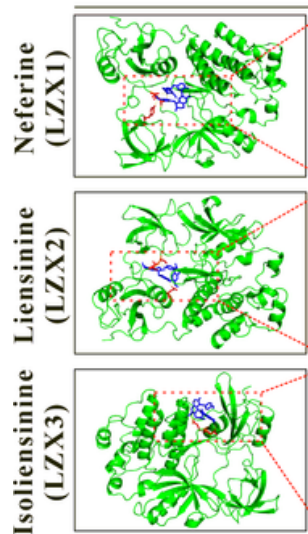

SRC

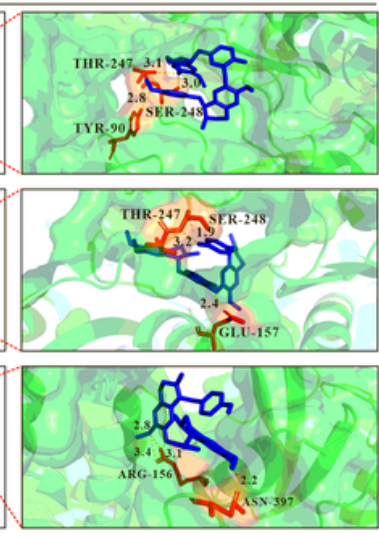

PIM1
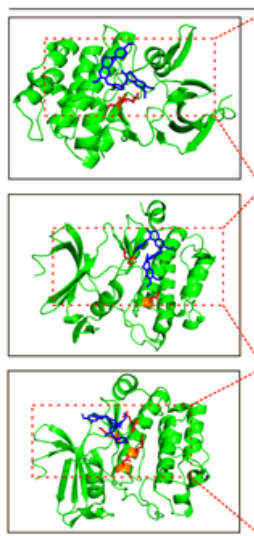
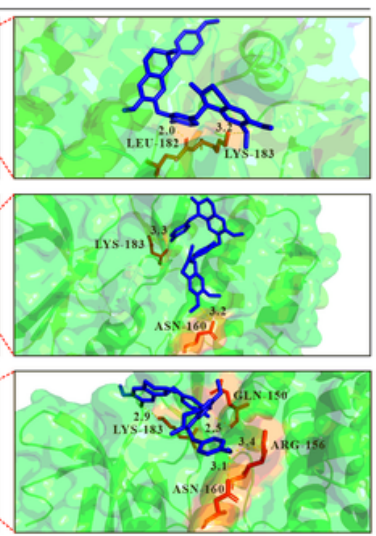

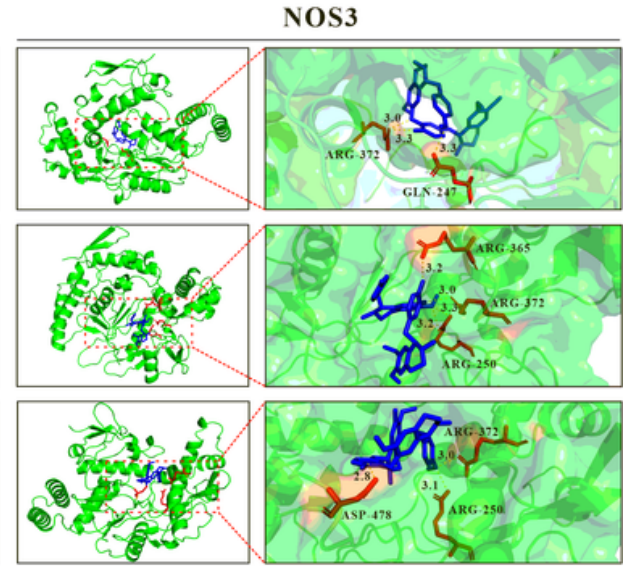

Figure 7

Molecular docking results of core compounds and targets. (A) The interaction between 7 candidate core compounds and ten core targets was evaluated by AutoDock Vina. (B) The molecular docking pattern between the core compounds and targets.

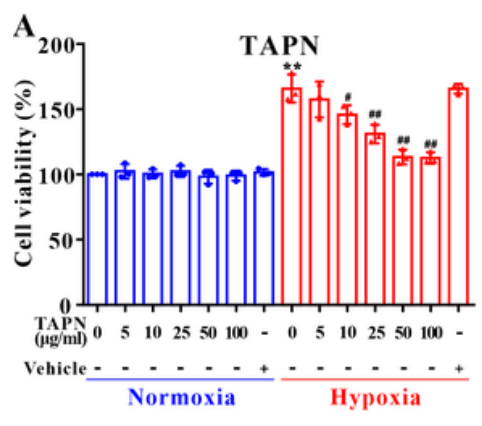

E

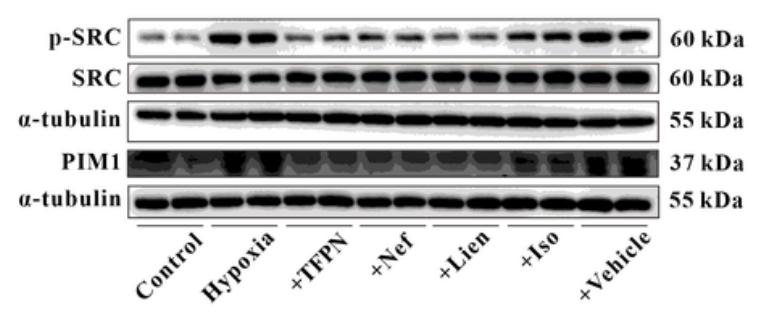

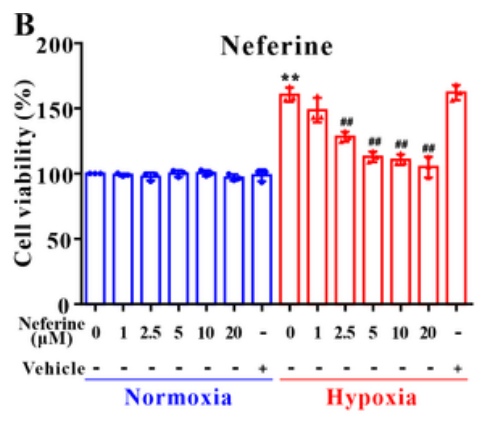

F

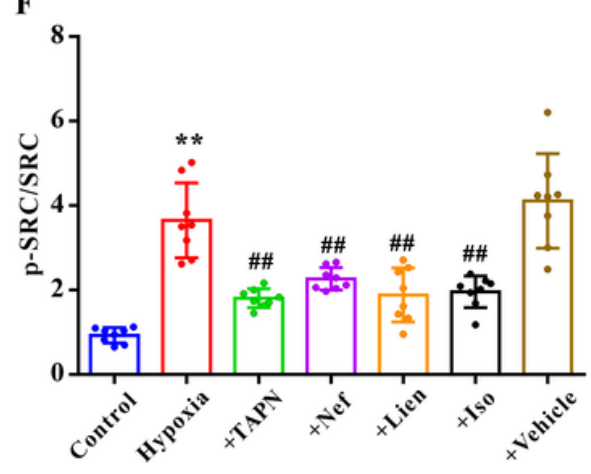

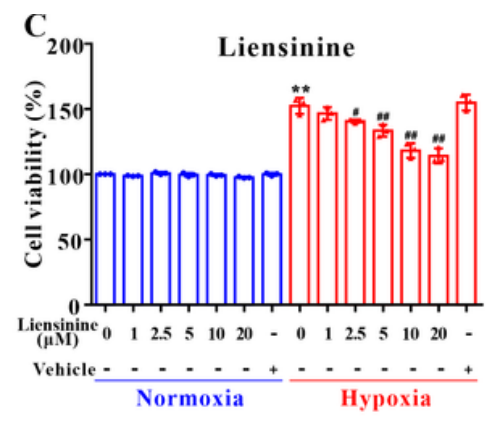
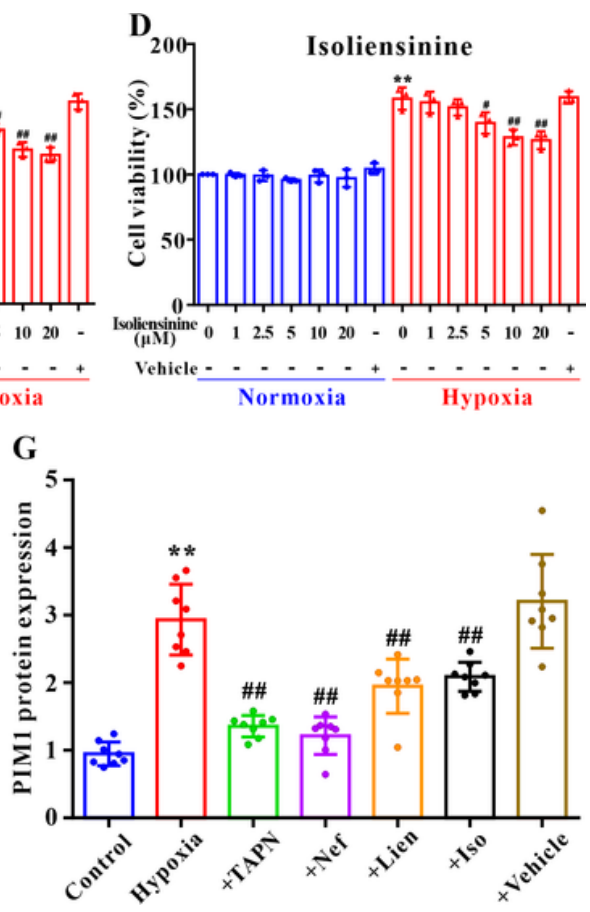


\section{Figure 8}

Plumula Nelumbinis attenuated hypoxia-induced proliferation of PASMCs through inhibition of the activation of SRC or PIM1. (A) Effects of TAPN, neferine, liensinine, and isoliensinine on the proliferation of PASMCs induced by hypoxia $(n=3)$. (B) The protein expression of p-SRC, SRC, and PIM1 in PASMCs ( $\mathrm{n}=8$ per group); Data are expressed as mean \pm SD. + TAPN: hypoxia + total alkaloids of Plumula Nelumbinis $(50 \mu \mathrm{g} / \mathrm{mL})$; +Nef: hypoxia + neferine $(5 \mu \mathrm{M})$; +Lien: hypoxia+ liensinine $(10 \mu \mathrm{M})$; +Iso: hypoxia+ isoliensinine $(10 \mu \mathrm{M}){ }^{* *} P<0.01$ vs Control; ${ }^{\#} P<0.05,{ }^{\# \#} P<0.01$ vs Hypoxia. 